\title{
La investigación científico-social: Un debate epistemológico desde la educación
}

\section{The Social-Scientific Research: An Epistemological Discussion from Education}

\author{
Omar Mejía Pérez ${ }^{1}$ \\ Centro de Estudios Superiores de México \\ Universidad Autónoma de Fresnillo \\ Cuernavaca, Morelos, México \\ klvita@hotmail.com
}

Recibido 27 de julio de 2011 • Aceptado 26 de agosto de 2011

\begin{abstract}
Resumen. El debate epistemológico de la investigación científico-social durante años ha consistido en confrontar los métodos, tratando de hacer valer más uno que el otro a partir de la gran teoría que lo soporta. Aunque propuestas más recientes recuperan métodos híbridos o planteamientos epistemológicos más diversos, la postura de la dualidad ha prevalecido. Descriptiva o explicativa; fenomenológica o positivista; clásica o emergente; los paradigmas de investigación son la manera en cómo tenemos acceso a la realidad investigada y así obtener principios y respuestas. Ello define los métodos. Aún cuando es posible agrupar y categorizar estos métodos en cuantitativos y cualitativos, el entramado epistemológico que establecen las estructuras de cualquier metodología permitirá el abordaje de un fenómeno mejor que otro, sin que esto pondere necesariamente alguno de manera definitoria. Dentro de la educación, tanto métodos cuantitativos como cualitativos han ayudado a abordar fenómenos de tal manera que han producido resultados favorables para el bienestar del quehacer educativo. Este ensayo, producto de una revisión bibliográfica y una trayectoria investigativa y educativa, pretende esclarecer el complejo panorama inicial de los investigadores educativos novatos antes de revisar y recuperar una postura epistemológica.
\end{abstract}

Palabras claves. Investigación, cientificismo-social, método, paradigma.

Abstract. The epistemological debate of social-scientific research has been, for years, to compare the methods used, trying to determine which one is more valuable, according to its theoretical basis. Although more recent proposals use hybrid methods or more diverse epistemological approaches, duality has prevailed. Descriptive or explanatory; phenomenology or positivist; classical or emergent; research paradigms are our way to access to the reality studied and obtain principles and answers. This defines the methods. Even when, it is possible to group and categorize these methods as quantitative and qualitative, the epistemological framework established by the structures of any methodology will allow approaching a phenomenon better than other, although, this does not necessarily make it definitely more valuable. Both quantitative and qualitative methods, in education, have helped to address phenomena, which has produced favorable results to benefit the education activity. This trial, which is the result of a literature review and a career in research and education, aims at clarifying the complex initial overview of new educational researchers, instead of reviewing an epistemological position.

Keywords. Research, social-scientism, method, paradigm.

\footnotetext{
Maestro en educación por la Universidad Autónoma de Fresnillo, México. Es doctorando por el Centro de Estudios Superiores de México en coordinación con la Universidad Autónoma de Fresnillo en el Doctorado en Educación. Es docente en la Universidad Autónoma de Morelos, en la Universidad del Valle de México y en el Colegio de Bachilleres del Estado de Morelos. Ha laborado para el Instituto de Educación Básica del Estado de Morelos, el Centro Morelense de las Artes, la Universidad de la Cuenca de Morelos y el Centro de Estudios Superiores de México. Actualmente está por concluir su trabajo de tesis doctoral.
} 


\section{Introducción}

La investigación social, en la actualidad, alude a un sinfín de concepciones y autores que en materia escolar generan demasiadas confusiones al momento de intentar desarrollar un proyecto de investigación. En muchas escuelas en México en donde se realizan estudios de posgrado en el campo pedagógico, la premisa inicial de toda línea investigativa radica en la selección de un método o en la tendencia hacia una metodología. Este suceso, fundamentalmente, complica la inserción efectiva de los educandos en la investigación real, pues los limita en la concepción epistemológica misma del acto de investigar cuando no se sientan las bases teóricas necesarias desde un principio del excéntrico y amplio panorama del quehacer investigativo, y simplemente se comienza por el análisis del problema y la elección del método, sin antes establecer los referentes filosóficos de una investigación y las amplia gama de posibilidades para desarrollarla.

Entender el proceso de investigación social como un simple mecanismo biparadigmático es una postura escueta e ineficiente. Por un lado, la actual postura clásica del positivismo ha sido alcanzada por un enfoque subjetivista-interpretativo que ha generado la metodología cualitativa y ha posibilitado estudios más detallados y descriptivos. Por otro lado, la ciencia misma que se engendra en la investigación y a su vez la faculta para llevarla a cabo rigurosamente ha entablado nuevas visiones regenerativas de su propia concepción gracias a los avances tecnológicos que han permitido nuevos descubrimientos y conocimientos, y han hecho de la ciencia ortodoxa una "nueva ciencia" (Alonso, 2008, p. 113) revolucionada, dinámica y compleja. Todo esto ha llevado a la investigación social -y en este caso educativa- a la amplitud que los fenómenos sociales-científicos requieren para su estudio y atención.

Queda ya anulado el discurso de la investigación como un proceso mecánico, unívoco e inflexible. El posmodernismo ha abierto las puertas a un mundo de nuevas concepciones investigativas propuestas por muchos autores que, basadas en la ciencia y la metodología correspondiente, han develado nuevas maneras de entender y atender la investigación. Este trabajo pretende entretejer un panorama más claro, en términos conceptuales, de lo que significa la investigación científico-social hoy en día desde un perfil educativo.

Esperemos, pues, que este escrito sirva para desenmarañar cabos sueltos, esclarecer dudas y complementar conceptos y argumentos para aquellos que vayan a realizar o se encuentren desarrollando una investigación social dentro del amplio campo de la educación.

Infinidad de teóricos se han dado a la tarea de proponer concepciones, paradigmas y métodos investigativos que sirvan de guía para trabajos científicos tanto naturales como sociales, incluidos en ellos los educativos. Comenzando por entender que la investigación es un proceso arduo y partiendo de la idea de Rojas, entendemos que es "(...) un conjunto de pasos y etapas que deben seguirse de manera mecánica para alcanzar sin mayor esfuerzo y creatividad la verdad científica" (2001, p. 65). Es necesario valorar este concepto, lejos de enfrascarnos en una discusión sobre la “(..) manera mecánica [y] (...) alcanzar sin mayor esfuerzo (...) la verdad científica” (2001, p. 65). Resulta imprescindible situarse dentro de un marco contextual canónico de reglas para obtener resultados válidos y fiables, que permitan el análisis de fenómenos sociales en su carácter histórico y tener la posibilidad de derivar de ellos veracidades científicas. Aunque como ahondaremos más adelante, estas verdades dentro de la investigación social no necesariamente deben llegar a postularse en axiomas sociales. 
Es importante insistir, en un primer momento, en la investigación como un proceso metodológico para enunciar propuestas resultantes, sin establecer aún ninguna diferencia de aplicabilidad tanto en la naturaleza como en el ámbito social, para posteriormente, poder clarificar con mayor rigor las condiciones que se establecen en los distintos escenariosy fenómenos y su metodología de estudio.

En una primera instancia, García (1994) define que la palabra investigación proviene de investigatio, que quiere decir rastrear, seguir una senda para descubrir algo desconocido por medio de un vestigium. Agrega que la investigación es un quehacer ni más ni menos mediante el cual se construye ciencia. Kerlinger (1975, p. 11; en Hernández, 1991, p. 15) define que la investigación científica es "sistemática, controlada, empírica, y crítica, de preposiciones hipotéticas sobre las presumidas relaciones entre fenómenos naturales". De esta manera, podemos entender la relación causal de un trabajo científico de investigación y sus resultados fehacientes en sus entornos posibles, tanto naturales como sociales. Hernández (1991), en la introducción de Metodología de la investigación, señala que la investigación científica es rigurosa y cuidadosamente realizada; que produce conocimiento y teoría (básica), y puede resolver problemas prácticos (aplicada); además la entiende como un proceso dinámico, cambiante y continuo, compuesto por etapas las cuales se derivan unas de otras.

Una investigación precisa en sí misma constituye una manera particular de analizar e interpretar la realidad. Un sujeto en calidad de investigador es un ente activo, sensorial y crítico desde su propia praxis; debe buscar la verdad y más aún la transformación factible de la realidad que asume. Además de entender la realidad desde su entorno teórico y práctico, el investigador para su optimización debe poseer cualidades de caza: sigiloso, observador, buen escucha, perseverante y curioso. "(...) El mero hecho de interpelar la realidad sobre la que se va a actuar y obtener datos sobre ella, sitúa al colectivo, equipo o investigador en una posición de escucha, que es condición imprescindible para cualquier proceso de intervención y transformación social" (Cembranos, Montesinos y Bustelo, 1989, p. 23).

En el ámbito educativo, la investigación se ciñe en fenómenos del quehacer pedagógico, andragógico, o bien, áreas humanas que trastocan los procesos formativos (política, economía, medicina, entre otras); aunque una verdad de Perogrullo es que dentro de la educación coinciden demasiadas disciplinas, tanto científicas como acientíficas, se vuelve un campo sumamente complicado para su estudio. El material investigativo está situado en las prácticas docentes, en los comportamientos y aprendizajes de los alumnos, en los espacios escolares diversos, en los lineamientos y determinaciones administrativas y directivas, en los currículos, en las políticas internas y externas, en las funciones escolares y sus agentes: es un quehacer inacabado e inacabable.

Muchos teóricos promueven una diferencia imperante en el estudio de las ciencias naturales y las sociales. Bordieu (1975, en Baranger, 2004) por su parte, postulaba que los errores de la sociología se enraízan en una falsa representación de la epistemología de las ciencias naturales. Los principios que han regido al positivismo ortodoxo en la actualidad han perdido mucha fuerza desde las nuevas posturas posmodernistas que entraman una nueva ciencia, por lo que la investigación, basada exclusivamente en el positivismo, ha dejado de ser la única alternativa investigativa.

Es precisamente el discurso sociológico el que “(...) abarca formas de debate más generalizadas que las discusiones científicas ordinarias. Mientras que estas se apoyan en evidencias empíricas específicas y en la lógica inductivo-deductiva, el discurso de las ciencias sociales se centra en el proceso de razonamiento más que en los resultados de la experiencia 
inmediata" (Alonso, 2008, p. 8). Ante esta aseveración, es necesario establecer las premisas relevantes de los métodos de investigación social frente a los axiomas del método científico baconiano. Estos métodos promueven, ante todo, una formulación de postulados a partir del análisis de la realidad fenoménica y su interpretación enmarcada en la teoría epistemológica adoptada. Cuando la ciencia pura ha defendido canónicamente un proceso hipotético-comprobable, los métodos de investigación social deben atender la realidad a partir de la experiencia y la interrelación intersubjetiva en contextos específicos, sin menoscabar el rigor metodológico que se establece dentro de los procesos de recolección de datos, construcción y piloteo de instrumentos, interpretación y categorización de elementos y variables, y análisis de resultados; con ello será posible conseguir algún nivel de generalización con variables y factores distintos y resultados simplemente conjeturables más no necesariamente repetibles. Si bien es cierto que la investigación debe contribuir a entender patrones de comportamiento y fenómenos generales, la investigación social no pretende establecer leyes de estructuras conductuales, sino más bien esquemas generales propiamente para el análisis sociológico-antropológico (o psicológico-filosófico desde una postura más amplia). Dentro de la educación, una de las necesidades investigativas básicas es el hacer notar fenómenos conmensurables que como generalidad repercutan en el desarrollo cognitivo de los sujetos aprendices y, con ello, en la evolución de las sociedades.

García (1972), en Alonso (2008), comenta que uno de los mitos más peligrosos en las ciencias sociales es creer que la teoría científico-social es universal y ahistórica. Para Alonso como para García, la ciencia social requiere de un esfuerzo mayor de abstracción y razón, en tanto, la naturaleza, un mero entendimiento de procesos y factores fenoménicos repetibles y sus leyes explicativas.

Es importante destacar que la divergencia explicitada en ambos fenómenos -sociales y naturales- radica en el planteamiento del fenómeno mismo. Un fenómeno natural que cumple con características bien definidas carece de ambigüedad al ser tratado como un evento aislado de estudio. Esto es posible al establecer modelos científicos que predispongan, al investigador, a analizar contextos herméticos con limitadas incidencias inesperadas. Los fenómenos sociales desde un inicio tienen la calidad de subjetividad y objetividad, de acuerdo con el enfoque planteado. Sus características obedecen al contexto histórico: cultura y genética de razonamiento, que desembocan en la estructura del fenómeno investigativo.

\section{Los enfoques epistemológicos en la investigación social}

Algunos autores (Rodríguez, Gil y García, 1996) señalan diversos enfoques epistemológicos para acercarse al conocimiento mediante el proceso investigativo social: (positivismo, materialismo, fenomenología, neo-marxismo, crítico, pos-estructural, por citar algunos). Otros (Rojas, 2001; Taylor y Bogdan, 1987) agrupan los enfoques y los hacen converger en una división simple bipartita -fenomenología/materialismo histórico vs positivismo-. Estas posturas refieren a un paradigma epistemológico de construcción de teoría establecido en una forma de entender la realidad. La manera de acceder al conocimiento de esta realidad está definida por las concepciones epistemológicas iniciales que dan pauta a paradigmas de investigación social.

Valles (1999) esboza una clasificación de la investigación social más abundante y más compleja. Propone las versiones de los dos, tres y cuatro paradigmas; en la primera, la de 
los dos paradigmas, ambos son contrapuestos, uno lo podremos catalogar como clásico y el otro como emergente. El primero de estos “(...) asume la existencia de una sola realidad objetiva averiguable a través de los cinco sentidos, sujeta a las leyes universales de la ciencia, y manipulable mediante procesos lógicos (...) [mientras que el otro, asume la existencia de] realidades múltiples, con diferencias entre ellas que no pueden resolverse a través de procesos racionales o aumentando los tamaños muestrales" (Erlandson y otros, 1993, 14). Este enfoque biparadigmático supone, como habrá de entenderse, la existencia de dos posibles modos de abordar un hecho investigativo. Uno proveniente del positivismo clásico, adaptado de los fenómenos naturales; el otro, como una respuesta alterna a este que pone de relieve la disparidad analítica de fenómenos naturales y sociales.

Este enfoque, que deviene directamente de los métodos cuantitativos y cualitativos de investigación, bifurca las líneas investigativas y señala las diferentes propiedades de los fenómenos, advirtiendo, por un lado, la objetividad de las situaciones naturales y su comportamiento repetible y predictible, y por otro, la complejidad psciosocial que demarcan los hechos del ser humano en sociedad.

En la propuesta de los tres paradigmas, Valles (1999, p. 55), basado en Crabtree y Miller (1992, p. 8), -y estos apoyados a su vez en Habermas (1968)-, identifica a los tres paradigmas como el de "a) (...) la indagación materialista, b) (...) constructivista y c) (...) crítica o ecológica". A diferencia de la versión de dos paradigmas, en esta se separa la constructivista de la crítica como una propuesta de características muy distintas.

De acuerdo con esta concepción, la indagación materialista se define en una lógica más bien positivista, basada prácticamente en el planteamiento del problema, revisión de la bibliografía, formulación de hipótesis, diseño, instrumentación, muestreo, recogida de datos, análisis de resultados y confrontación de hipótesis: el método científico baconiano.

La indagación constructivista se basa en el conocimiento que nos ayuda a establecer el equilibrio cultural, de comunicación y de construcción de significados simbólicos; mantiene un diseño muy abierto a la invención, al análisis y a la interpretación: el interaccionismo simbólico.

La indagación crítica o ecológica establece las relaciones sociales como punto de partida, la distribución del poder y las desigualdades asociadas. Se adecua, uniformemente, al sistema político y a las necesidades del planteamiento de los problemas, mientras las relaciones sociales modifican los procesos internos: el materialismo dialéctico.

Otros autores (Babbie, 1979; Selltiz, Jahoda, Deutsch y Cook, 1965; en Hernández, 1991) clasifican los tipos de investigación de manera tripartita en exploratorios, descriptivos y explicativos. La forma en que los teóricos proponen paradigmas, alude a la temporalidad y características culturales propiamente de la época y los hallazgos que prevalezcan y ocupen un lugar en las sociedades científicas.

Finalmente, en la última versión, los enfoques se agrupan en cuatro paradigmas: positivismo, postpositivismo, teoría crítica y enfoques afines, y constructivismo. Valles (1999) se basa en Guba y Lincoln (1994) para esta clasificación, y demarca una distinción evolutiva entre el positivismo y el postpositivismo, así como un agrupación de varios enfoques -materialismo, neomarxismo, feminismo- dentro de la teoría crítica. También señala que el constructivismo es un relativismo derivado de realidades construidas en contextos concretos, por lo que es necesario categorizarlo fuera de los otros. 
El esfuerzo por agrupar paradigmas en dos o más corrientes epistémicas tiene como finalidad objetivar criterios tanto ontológicos como epistemológicos y brindar una visión más concreta de la manera de estructurar la realidad e investigar fenómenos sociales.

\section{De la postura biparadigmática}

Rojas, en su libro Investigación social: teoría y praxis postula que “(..) los principios y reglas metodológicas así como el uso de las técnicas e instrumentos de recolección y análisis de datos se ubican dentro de una determinada perspectiva teórica (materialismo histórico, positivismo y sus variantes: funcionalismo y conductismo, etcétera). Por lo tanto, la metodología que se utilice para realizar una investigación social estará en función del tipo de enfoque teórico que se elija para el desarrollo de la investigación (...)" (2001, p. 68). Como hemos señalado, la postura epistemológica definirá la metodología, es decir “(...) el modo en que enfocamos los problemas y buscamos respuestas (...)" (Taylor y Bogdan, 1987, p. 15). Este, a su vez, definirá las características subsecuentes de investigación; pero entendamos que la postura inicial fundamentará concretamente los cimientos de nuestros postulados hipotéticos. La genealogía histórica de los dos paradigmas ha definido la investigación social de manera muy sólida y contundente en sus resultados. Esta manera, ha favorecido enormemente la investigación social y la ha simplificado. De cualquier modo, esta postura dual es igualmente entendida desde distintas formas.

Taylor y Bogdan (1987, pp. 15-16) hablan de positivismo y fenomenología como las dos corrientes teóricas generales de la investigación: “(...) los positivistas buscan los hechos o causas de los fenómenos sociales con independencia de los estados subjetivos de los individuos [cursiva añadida] (...) el fenomenólogo quiere entender los fenómenos sociales desde la propia perspectiva del actor [cursiva añadida] (...). Examina el modo en que se experimenta el mundo. La realidad que importa es lo que las personas perciben como importante". Se señalan estas dos posturas contrapuestas de igual modo como el paradigma positivista, prevaleciente, clásico, racionalista, o desde el otro extremo, el paradigma emergente, alternativo, naturalista, constructivista, interpretativista. Para la educación, ambos paradigmas han contribuido a desarrollar investigaciones y teorías importantes, fundamentalmente para explicar desde un enfoque interpretativo los modelos de aprendizaje de los alumnos o, desde el positivismo, el funcionamiento de un modelo didáctico experimental, por mencionar algunos.

Indistintamente de la nomenclatura formal, para los positivistas el hecho es encontrar la generalidad de las situaciones de estudio; encontrar la objetividad en los fenómenos. Durkheim (1938 en Casas, 1998) afirmaba que el científico social tiene que considerar los fenómenos sociales o hechos como "cosas", y que estas ejercen una influencia externa sobre los sujetos.

Para la otra postura -materialismo dialéctico, fenomenología y demás-, la interpretación se vuelve el eje vertebral de la investigación, por tanto, el sustento teórico del investigador será su fundamento más poderoso y de él devendrán sus teorizaciones y conjeturas. Jack Douglas (1970) pensaba que las fuerzas que mueven a los seres humanos no nos convierten en cuerpos de materia inerte, sino materia significativa, animada, con ideas, sentimientos y motivos internos. Los fenomenólogos -según Max Weber (1968)- luchan por el verstehen -que significa comprensiónesto es "(...) comprensión en un nivel personal de los motivos y creencias que están detrás de las acciones de la gente" (Taylor y Bogdan, 1987, p. 16). 
Algunos otros autores conciben la investigación como descriptiva o explicativa; la primera tiene como objetivo "(...) llegar a conocer las situaciones, costumbres y actitudes predominantes a través de la descripción exacta de las actividades, objetos, procesos y personas (...)" (Van Dalen y Meyer, 1981, pp. 52-53). La investigación explicativa o experimental se encarga de buscar el porqué de los hechos mediante el establecimiento de relaciones causa-efecto. En este sentido, los estudios explicativos pueden ocuparse tanto de la determinación de las causas (investigación postfacto), como de los efectos (investigación experimental), mediante la prueba de hipótesis, es decir, cuantitativamente. Es así como podemos relacionar la bifurcación metodológica hacia una tendencia cuantitativa o cualitativa.

Es preciso entender que los fenómenos de estudio poseen características muy particulares. Los resultados de una investigación no tan solo están definidos por el buen o mal trato de sus variables, actores o circunstancias, o por la elección del método o el diseño y aplicación de sus instrumentos. Está inicialmente encapsulado en la concepción de la realidad del mundo. No condenamos al paradigma deductivo por su feroz tenacidad y poca flexibilidad procedimental, ni tampoco al interpretativo por su subjetividad. La intención es ceñir los horizontes a una realidad óntica y analizar los fenómenos desde la perspectiva que demuestre mayores expectativas epistemológicas.

\section{El enfoque cualitativo y cuantitativo en las ciencias sociales}

Conviene explicar que los paradigmas epistemológicos, como hemos expuesto a lo largo de nuestro discurso, definen la manera en cómo enfrentamos los hechos y problemas para indagar sus principios y posibles soluciones. Esto precisa el método, es decir, el nivel más alto de abstracción que "(...) designa los procedimientos lógicos, inherentes a toda investigación científica y, por tanto, independientes de todo contenido concreto, que debe seguir el investigador para obtener la verdad y verificarla (...)" (Alonso, 2008, p. 19). Entonces, el método descifra la manera de estructurar las técnicas, instrumentos y procesos deductivos e interpretativos dentro del escenario investigativo y la interacción de los actores.

Desde la perspectiva más simplista, concebimos que, de alguna u otra forma, las investigaciones sociales pueden abordarse desde dos perspectivas metodológicas base: la primera, atendiendo con más rigor el método científico riguroso y la segunda, usando un método interpretativo más subjetivo: “(...) en la investigación se enfatizan explícitamente dos de las categorías de más amplio uso en todo proceso científico: lo cualitativo y lo cuantitativo (...)" (Laureiro y Fernández 1998, p. 2). Una mera tendencia más hacia la postura interpretativa o hacia lo deductiva.

García (1994, p. 28) propone que “(...) el estudio cuantitativo ofrece con más precisión la magnitud de las relaciones (...) el estudio cualitativo concluye en afirmaciones `más ambiguas’ pero con mayor atención a la influencia y al sentido de los distintos factores que inciden en una determinada actuación pedagógica" (Firestone, 1987, p. 19). Un método cuantitativo será utilizado para estudiar fenómenos de carácter más estadístico, que requieran de experimentación, de resultados más tangibles, numéricos, de clasificación, de explicaciones más concretas, de graficación y objetivación. Por ejemplo, ante el problema de la reprobación en el estado de Morelos, sería conveniente implementar algún método cuantitativo para esbozar características, rasgos y factores repetitivos en distintas 
muestras con variables similares. Por otro lado, un método cualitativo será útil para fenómenos que requieran de mayor atención a sus cualidades, que necesiten ser más descriptivos; que se entiendan por su comportamiento complejo y no por su composición estructural; que su naturaleza sea más reflexiva que repetitiva, rasgos sobresalientes en los fenómenos sociológicos. Un ejemplo podría ser el de la cultura docente apática al autodidactismo en México. Aunque las dimensiones de la evaluación favorecen a uno u otro método -las investigaciones a gran escala favorecen el método cuantitativo y las de menor escala, las de cualitativo- no es una determinante para las investigaciones. Si bien es cierto que un fenómeno educativo puede investigarse mediante alguna metodología mejor que otra, también es cierto que los fines investigativos determinan con mayor precisión la postura investigativa. Finalmente los estudios, tanto cuantitativos como cualitativos, se realizan de manera separada y producen resultados con diferente clase de información "(...) mas pueden referirse a una misma realidad óntica" (García, 1994, p. 28).

Goetz y LeCompte (1988) postulan diversos modos suposicionales de los hechos al enfocar la realidad y su explicación de distintas formas. Tales modos pueden categorizarse en cuatro dimensiones en relación con las estrategias analíticas: inductivo-deductiva, generativo-verificativa, constructivo-enumerativa y subjetivo-objetiva. Estos modos son contrapuestos y pretenden analizar y explicitar los fenómenos desde el enfoque conforme a su naturaleza.

Estos modos suposicionales comparativos tienen un orden entre sí que categoriza al método distintivo de investigación, ya sea cuantitativo o cualitativo. "Por investigación cuantitativa se entiende un estudio deductivo, verificativo, enumerativo y objetivo; la investigación cualitativa denotaría procesos inductivos, generativos, constructivos y subjetivos" (Goetz y LeCompte, 1988, p. 32).

Uno de los métodos de investigación cualitativa más utilizado en materia educativa es la etnografía educativa, que recupera la interpretación de la acción significativa, el entramado cultural y los instrumentos de significación en los escenarios educativos (Bertely, 2000, p. 30-36). Otro de los métodos cuantitativos más utilizados es el método hipotético-deductivo, que consiste en confrontar una hipótesis inicial con las variables de la realidad experimental de un determinado escenario educativo y verificar sus principios de veracidad. Finalmente, ambas posturas pueden solventar la investigación de un fenómeno educativo, siempre y cuando se establezca a priori, con argumentos válidos, la necesidad de hacerlo de un modo o de otro para demostrar o explicitar un problema educativo.

\section{Conclusiones}

No existe, desde nuestra perspectiva, algún argumento suficientemente contundente que favorezca notoriamente determinada metodología. Aun cuando incluso nosotros nos inclinemos por la metodología cualitativa dentro de la investigación educativa, por destacar más la esencia conductual compleja del ser humano, no desacreditamos la utilidad, funcionalidad e importancia de los métodos cuantitativos que pueden ser capaces de ofrecer panoramas muy claros del comportamiento de fenómenos generales o tendenciosos.

Es preciso entender, al iniciar cualquier estudio educativo -e incluso en cualquier campo social-, que como investigador, principalmente novato, debo comenzar por esclarecer mi horizonte primario y hacer un gran esfuerzo para asumirme dentro de alguna postura epistemológica, en donde 
pueda percibir, analizar y registrar los hechos de la realidad conforme a mí empírea y conocimiento factual, respondiendo a la manera en cómo entiendo la construcción del conocimiento y, con ello, definir mi marco de referencia teórico, mi postura metodológica y mi técnica investigativa.

Independientemente de que existan infinidad de autores, paradigmas y métodos investigativos, lo crucial dentro de una investigación es llevar a cabo un análisis preliminar de nuestra postura epistemológica con respecto al fenómeno y desarrollar un esquema de estudio detallado; ello declinará por sí mismo una metodología, y esto a su vez las formas precisas de efectuarla.

\section{Referencias bibliográficas}

Alonso, J. A. (2008). Metodología. México: Limusa.

Baranger, D. (2004). Los oficios del sociólogo (Cap. II) En D. Baranger (Aut.), Epistemología y metodología en la obra de Pierre Bordieu (pp. 59-87). Buenos Aires: Prometeo.

Bertely, M. (2000). Conociendo nuestras escuelas: Un acercamiento etnográfico a la cultura escolar. México: Paidós.

Casas, M. C. (1998). La fundamentación de una ciencia social autónoma. El pensamiento de Durkheim: entre el mundo empírico y el simbólico. En R. Reséndiz (Coord.), El pensamiento sociológico clásico (107-153). México: UNAM.

Cembranos, F., Montesinos, D. H. y Bustelo, M. (1989). El análisis de la realidad (Cap. II). En F. Cembranos, D. H. Montesinos y M. Bustelo (aut.), La animación sociocultural. Una propuesta metodológica (pp. 23-55). Madrid: Popular.

García, V. (1994). La investigación pedagógica y el principio de distinción y complementariedad. En V. García (Coord.), Problemas y métodos de investigación en educación personalizada (pp. 21-38). Madrid: Rialp.

Goetz, J. P y LeComte, M. D. (1988). Etnografía y diseño cualitativo en investigación educativa. Madrid: Morata.

Hernández, R. (1991). Metodología de la investigación (3 a ed.). México: McGraw-Hill

Laureiro, I. y Fernández R. (mayo, 2006). Resumen de La dialéctica materialista Vs. Los "Paradigmas" de investigación cualitativa y cuantitativa. Ponencia presentada en la III Conferencia Internacional "La obra de Carlos Marx y los desafíos del siglo XXI". La Habana, Cuba.

Rodríguez, Gil y García (1996). Tradición y enfoques en la investigación cualitativa y métodos de investigación cualitativa (Cap. I y II). En G. Rodríguez, J. Gil y García, E. (Aut.), Metodología de la investigación cualitativa (pp. 1-35). Aljibe: Málaga 
URL: http://www.una.ac.cr/educare

Rojas, R. (2001). Formación de investigadores educativos. Una propuesta de investigación (10 $\left.{ }^{\mathrm{a}} \mathrm{ed}.\right)$. México: Plaza y Valdés

Taylor, S. J. y Bogdan, R. (1987). Introducción a los métodos cualitativos de investigación. Barcelona: Paidós.

Valles, M. S. (1999). Técnicas cualitativas de investigación social. Reflexión metodológica y práctica profesional. Madrid: Síntesis.

Van Dalen, D. B. y Meyer, W. J. (1988). Manual de técnica de la investigación educacional. Argentina: Paidós. 